CLINICAL STUDY

\title{
ADMA concentration changes across the menstrual cycle and during oral contraceptive use: the Cardiovascular Risk in Young Finns Study
}

Pirjo Valtonen ${ }^{1}$, Kari Punnonen ${ }^{1}$, Heli Saarelainen ${ }^{2}$, Nonna Heiskanen ${ }^{2}$, Olli T Raitakari ${ }^{3}$, Markus Juonala ${ }^{4}$, Jorma S A Viikari ${ }^{4}$, Georg Alfthan ${ }^{5}$, Mika Kähönen ${ }^{6}$, Reijo Laaksonen ${ }^{7}$, Tiina Lyyra-Laitinen ${ }^{8}$, Tomi Laitinen ${ }^{8}$ and Seppo Heinonen ${ }^{2}$

Departments of ${ }^{1}$ Clinical Chemistry and ${ }^{2}$ Obstetrics and Gynaecology, Kuopio University Hospital and University of Kuopio, FIN-70210 Kuopio, Finland, Departments of ${ }^{3}$ Clinical Physiology and ${ }^{4}$ Internal Medicine, University of Turku, FIN-20520 Turku, Finland, ${ }^{5}$ Disease Risk Unit, National Institute of Health and Welfare, FIN-O0271 Helsinki, Finland, ${ }^{6}$ Department of Clinical Physiology, Tampere University Hospital and University of Tampere,

FIN-33520 Tampere, Finland, ${ }^{7}$ Laboratory of Atherosclerosis Genetics, Department of Clinical Chemistry, Medical School, Centre for Laboratory Medicine and University of Tampere, Tampere University Hospital, FIN-33520 Tampere, Finland and ${ }^{8}$ Department of Clinical Physiology and Nuclear Medicine, Kuopio University Hospital and University of Kuopio, FIN-70210 Kuopio, Finland

(Correspondence should be addressed to P Valtonen; Email: pirjo.valtonen@uku.fi)

\begin{abstract}
Objective: The aim of this study was to evaluate changes in the nitric oxide synthase inhibitor asymmetric dimethylarginine (ADMA) levels during different menstrual cycle phases in young adult women with or without oral contraceptive (OC) use.

Design and methods: The subjects $(n=1079)$ originated from a large population-based, prospective cohort study conducted in Finland. Plasma ADMA, symmetric dimethylarginine (SDMA), L-arginine, C-reactive protein, creatinine, and brachial artery flow-mediated dilatation (FMD) were measured. The use of OCs and menstrual cycle phase were determined from a questionnaire.

Results: In non-OC users, ADMA $(P=0.017)$, L-arginine $(P=0.002)$, and ADMA/SDMA ratio $(P<0.001)$ were significantly lower in the luteal phase than in the follicular phase of the menstrual cycle. Non-OC users also had significantly higher ADMA and SDMA concentrations $(P<0.001)$ and lower L-arginine concentrations $(P<0.001)$ compared to OC users of estrogen-containing pills. Progestin-only contraceptive pills (POPs) did not lower the ADMA level, but maintained it at the same level as in non-OC users. In OC users, there were no significant differences found in ADMA, FMD, or FMD \% across menstrual cycle, whereas brachial artery diameter was significantly more decreased in the luteal phase $(P=0.013)$ than in the follicular phase.

Conclusion: We observed that the circulating ADMA concentration varies across the menstrual cycle in young women not using OCs, and women on OCs displayed significantly lower circulating ADMA concentrations than non-OC users, though this was not the case with POP contraception.
\end{abstract}

European Journal of Endocrinology 162 259-265

\section{Introduction}

The menstrual cycle is regulated by hormonal cyclic changes. At the beginning of the follicular phase, estrogen and progesterone levels are low. The estrogen level starts to increase toward the end of the follicular phase, peaking during the surge of LH and FSH. The progesterone level is low during the follicular phase, but at the beginning of the luteal phase, it starts to increase. During most of the luteal phase, the progesterone and estrogen levels are high.

Estrogen can induce arterial vasodilation by activating endothelial nitric oxide (NO) synthase (NOS) (1). Cevik et al. (2) have suggested that estradiol may facilitate NO synthesis by reducing circulating levels of asymmetric dimethylarginine (ADMA), and this may exert protective effects on the vasculature. We and others have previously shown that the levels of ADMA, an endogenous competitive inhibitor of NOS, are inversely associated with flow-mediated brachial artery dilatation $(3,4)$, and ADMA has also been postulated to be a risk marker of atherosclerosis and endothelial dysfunction $(5,6)$, e.g. increased concentrations of ADMA have been reported in patients with hypertension, insulin resistance, hypercholesterolemia, and renal failure $(7,8)$. In addition, the concentration of ADMA has been shown to be elevated in patients with polycystic ovary syndrome (PCOS) (9) and complicated pregnancies (10). Symmetric dimethylarginine (SDMA), a stereoisomer of ADMA, cannot directly inhibit NOS, 
but it is able to indirectly reduce intracellular L-arginine availability and in this way limit NO synthesis (11). SDMA has been associated with renal dysfunction (12).

Recently, there has been an increasing interest in measuring ADMA in a variety of clinical settings. Oral contraceptives (OCs) are known to lower ADMA concentrations significantly in women with PCOS (9), and orally administered hormone replacement therapy (HRT) also has an effect on circulating ADMA levels (13-15). Our own studies and those of others have shown that during normal pregnancy, when there are high circulating estrogen levels, ADMA and SDMA concentrations are significantly decreased compared to the levels of non-pregnant women $(10,16,17)$. On the other hand, female sex hormones normally fluctuate according to the phases of the menstrual cycle, but as yet, the effects of menstrual cycle phases on ADMA concentrations have not been clarified.

The aim of the present study was to evaluate the changes in ADMA levels during different menstrual cycle phases in young adult women at the population level with or without OC use and also according to the type of OC. This study is a part of the Cardiovascular Risk in Young Finns Study (18).

\section{Subjects and methods}

\section{Subjects}

The subjects of the present study originated from the Cardiovascular Risk in Young Finns Study. This is an ongoing, prospective, five-center follow-up study of atherosclerosis risk factors in Finnish children and adolescents. The participants were randomly chosen from a national population register as described previously (18). At the beginning of the study in 1980, there were 3596 participants. In the follow-up study in 2001, 2283 young adults were studied in the age range from 24 to 39 years (19). In the present study, we selected women $(n=761)$ without hormone use and women $(n=318)$ who were using OCs. In non-OC users, $20(2.6 \%)$ subjects were on antihypertensive medication. In OC users, eight subjects $(2.5 \%)$ were on antihypertensive medication and one subject $(0.3 \%)$ was on lipid-lowering medication. The menstrual cycle phase at the sampling time and the use of OCs were obtained from the participants. The menstrual cycle was divided into four different phases: early follicular phase (1-7 days from the beginning of menstruation), late follicular phase (8-14 days from the beginning of menstruation), early luteal phase (15-19 days from the beginning of menstruation), and late luteal phase (20-40 days from the beginning of menstruation). Contraceptive types were divided into four categories: constant estrogen dose with low progestin dose $(n=110)$, constant estrogen dose with high progestin dose $(n=120)$, progestin alone $(n=10)$, and combination preparation with changing doses $(n=68)$.

The local ethics committee approved this study, and all patients provided written informed consent before participating.

\section{Procedures}

All blood samples were drawn after a 12-h overnight fast. Venous blood samples were drawn from each subject for laboratory assays and were centrifuged at $2000 \boldsymbol{g}$ for $10 \mathrm{~min}$. The serum was separated and stored frozen until analysis. Height, weight, and waist circumference were measured, and body mass index (BMI) was calculated (weight/height ${ }^{2}$ ).

\section{Measurement of plasma ADMA, SDMA and $L$-arginine concentrations}

Levels of serum L-arginine, ADMA, and its stereoisomer, SDMA, were determined by high performance liquid chromatography using precolumn derivatization with $o$-phthaldialdehyde according to the method described by Teerlink et al. (20) with modifications (4). Briefly, samples were purified by protein precipitation with trichloroacetic acid and by polymer solid-phase extraction cartridge. Monomethylarginine was used as an internal standard, and chromatographic separation was achieved with a Symmetry C18 column $(5 \mu \mathrm{m}$, $3.9 \times 150 \mathrm{~mm}$, Waters Corp., Milford, MA, USA). The analytes were detected by fluorescence at $\lambda^{\mathrm{ex}}=340 \mathrm{~nm}$ and $\lambda^{\mathrm{em}}=455 \mathrm{~nm}$. Precision (coefficient of variation, $\mathrm{CV})$ for a plasma pool $(n=77)$ for L-arginine, ADMA, and SDMA within series was $7.5,5.7$, and $6.5 \%$, and between series, it was $12.9,10.6$, and $12.1 \%$ respectively.

\section{Measurement of high-sensitive C-reactive protein and creatinine concentrations}

Plasma high-sensitive C-reactive protein (hsCRP) concentrations were analyzed by latex immunoturbidimetric assay (CRP-UL, Wako Chemicals GmbH, Neuss, Germany) by an automated analyzer (Olympus AU400, Tokyo, Japan). The detection limit was $0.06 \mathrm{mg} / \mathrm{l}$. The interassay CV $(n=168)$ were $3.3 \%(C R P 1.52 \mathrm{mg} / \mathrm{l})$ and $2.7 \%$ (CRP $2.51 \mathrm{mg} / \mathrm{l})$.

Serum creatinine concentrations were measured by standard photometric methods (Olympus Diagnostica GmbH, Hamburg, Germany), and the estimated glomerular filtration rate (GFR) was calculated by Cockcroft-Gault formula (21).

\section{Vascular ultrasound measurements}

Brachial artery flow-mediated dilatation (FMD) was measured by ultrasound according to the guidelines and as described earlier (19). Ultrasound studies were 
performed using Sequoia 512 mainframes (Acuson, Mountain View, CA, USA) with 14.0 MHz linear array transducers. The segment of the brachial artery above the antecubital crease was imaged in the longitudinal plane at rest and during reactive hyperemia, induced by a sphygmomanometer cuff, which was placed around the forearm, inflated to a pressure of $250 \mathrm{mmHg}$, and deflated after $4.5 \mathrm{~min}$. End-diastolic (incident with the $\mathrm{R}$-wave) arterial diameter was measured at rest (baseline) and at 40, 60, and $80 \mathrm{~s}$ after cuff release from 5-s ultrasound image sets. The vessel diameter response in scans after reactive hyperemia was expressed both as the absolute change in diameter (FMD) and as the percentage relative to the resting scan (FMD\%).

\section{Statistical analysis}

In the statistical analysis, results are presented as the mean \pm s.D. or S.E.M. or as median values. Comparisons between studied groups were performed with the one-way ANOVA test differences and between groups with $t$-test analysis. The correlations were calculated by the Pearson or Spearman correlation coefficient test. Log-transformed values were used for CRP and GFR because of a skewed distribution. A computer software program (SPSS 15.0 for Windows; SPSS Inc., Chicago, IL, USA) was used to analyze the data. A probability level $<0.05$ was considered statistically significant.

\section{Results}

\section{Effect of menstrual cycle}

The clinical characteristics of the study participants are presented in Table 1. The concentrations of ADMA and L-arginine varied significantly depending on the menstrual cycle phase. The ADMA level was significantly lower in the early luteal phase $(P=0.036)$ than in the early follicular phase (Fig. 1). When the early and late follicular and early and late luteal phase groups were pooled, then ADMA level was higher in the follicular phase than in the luteal phase of menstrual cycle $(0.635 \pm 0.144$ vs $0.609 \pm 0.147 \mu \mathrm{mol} / \mathrm{l}, P=0.017$, respectively; Fig. 2). The L-arginine concentration was lowest in the early luteal phase $(P=0.016$; Fig. 1$)$. SDMA and CRP concentrations or GFR exhibited no significant differences between menstrual cycle phases. ADMA/SDMA ratio, an index of the ADMA-degrading enzyme dimethylarginine dimethylaminohydrolase (DDAH) activity, varied significantly during the follicular and luteal phases $(P<0.001$; Fig. 2$)$, being at its lowest level in the early luteal phase. L-arginine/ADMA ratio, an indicator of NO production by NOS, did not vary significantly during the different menstrual cycle phases.

FMD was highest in the follicular phase (Fig. 1), although FMD, FMD\%, or brachial artery diameter did not differ significantly during the different phases of menstrual cycle.
Table 1 Clinical characteristics of women with and without oral contraceptive (OC) use. Values are presented as mean \pm s.D., and groups were compared by $t$-test $(P$ value).

\begin{tabular}{lccr}
\hline Characteristic & $\begin{array}{c}\text { Non-OC users } \\
(n=761)\end{array}$ & $\begin{array}{c}\text { OC users } \\
(n=318)\end{array}$ & $\boldsymbol{P}$ value \\
\hline Age (years) & $33 \pm 5$ & $30 \pm 5$ & $<0.001$ \\
Weight $(\mathrm{kg})$ & $67.6 \pm 13.9$ & $66.2 \pm 11.7$ & 0.085 \\
Height $(\mathrm{m})$ & $1.66 \pm 0.06$ & $1.67 \pm 0.06$ & 0.008 \\
Waist & $79.7 \pm 11.8$ & $76.9 \pm 9.8$ & $<0.001$ \\
$\quad$ circumference (cm) & & & \\
BMl & $24.6 \pm 4.8$ & $23.8 \pm 4.0$ & 0.004 \\
Systolic BP (mmHg) & $112 \pm 12$ & $115 \pm 13$ & 0.002 \\
Diastolic BP (mmHg) & $69 \pm 10$ & $70 \pm 8$ & 0.020 \\
GFR (ml/min) & $119.7 \pm 29.3$ & $115.8 \pm 26.5$ & 0.051 \\
hsCRP (mg/l) & $1.49 \pm 2.95$ & $3.86 \pm 6.44$ & $<0.001$ \\
ADMA ( $\mu \mathrm{mol} / \mathrm{l})$ & $0.624 \pm 0.149$ & $0.541 \pm 0.129$ & $<0.001$ \\
SDMA ( $\mu \mathrm{mol} / \mathrm{l})$ & $0.388 \pm 0.106$ & $0.368 \pm 0.092$ & 0.002 \\
L-arginine ( $\mu \mathrm{mol} / \mathrm{l})$ & $111.1 \pm 35.3$ & $129.2 \pm 41.7$ & $<0.001$ \\
L-arginine/ADMA ratio & $180.5 \pm 50.5$ & $243.6 \pm 72.6$ & $<0.001$ \\
ADMA/SDMA ratio & $1.65 \pm 0.30$ & $1.49 \pm 0.23$ & $<0.001$ \\
Brachial artery & $3.11 \pm 0.33$ & $3.08 \pm 0.32$ & 0.221 \\
$\quad$ diameter (mm) & & & \\
FMD (mm) & $0.27 \pm 0.13$ & $0.26 \pm 0.13$ & 0.734 \\
FMD (\%) & $8.69 \pm 4.47$ & $8.70 \pm 4.38$ & 0.980 \\
\hline
\end{tabular}

ADMA, asymmetric dimethylarginine; BMI, body mass index; BP, blood pressure; FMD, flow-mediated dilatation; GFR, glomerular filtration rate; hsCRP, high-sensitive C-reactive protein; SDMA, symmetric dimethylarginine.

\section{Effect of OC use}

ADMA $(P<0.001)$ and SDMA $(P=0.002)$ concentrations were significantly lower and L-arginine concentrations $(P<0.001)$ were higher in OC users than in non-OC users. L-arginine/ADMA ratio was higher $(P<0.001)$ and ADMA/SDMA ratio was lower $(P<0.001)$ in OC users than in non-OC users. The CRP concentration was 2.6-fold higher in OC users than in non-OC users. No significant differences were observed in GFR or FMD parameters between OC and non-OC users (Table 1). When examining the associations between parameters, CRP correlated significantly with GFR in both non-OC users and OC users $(r=0.404$, $P<0.001$ and $r=0.417, P<0.001$ respectively).

In OC users, there were no significant differences found in ADMA and ADMA-related parameters, CRP, or GFR between menstrual cycle phases. Furthermore, the studied FMD parameters did not differ significantly during the four phases of menstrual cycle in non-OC users or OC users. When the early and late follicular and early and late luteal phase groups were pooled, the brachial artery diameter was higher in the follicular phase than in the luteal phase $(3.13 \pm 0.31$ vs $3.03 \pm 0.33 \mathrm{~mm}, P=0.013$ respectively) in OC users.

Age and BMI displayed a significant difference between OC user and non-OC user groups. Furthermore, even when studied parameters were standardized according to age and BMI, this difference stayed significant between $\mathrm{OC}$ and non-OC groups. 

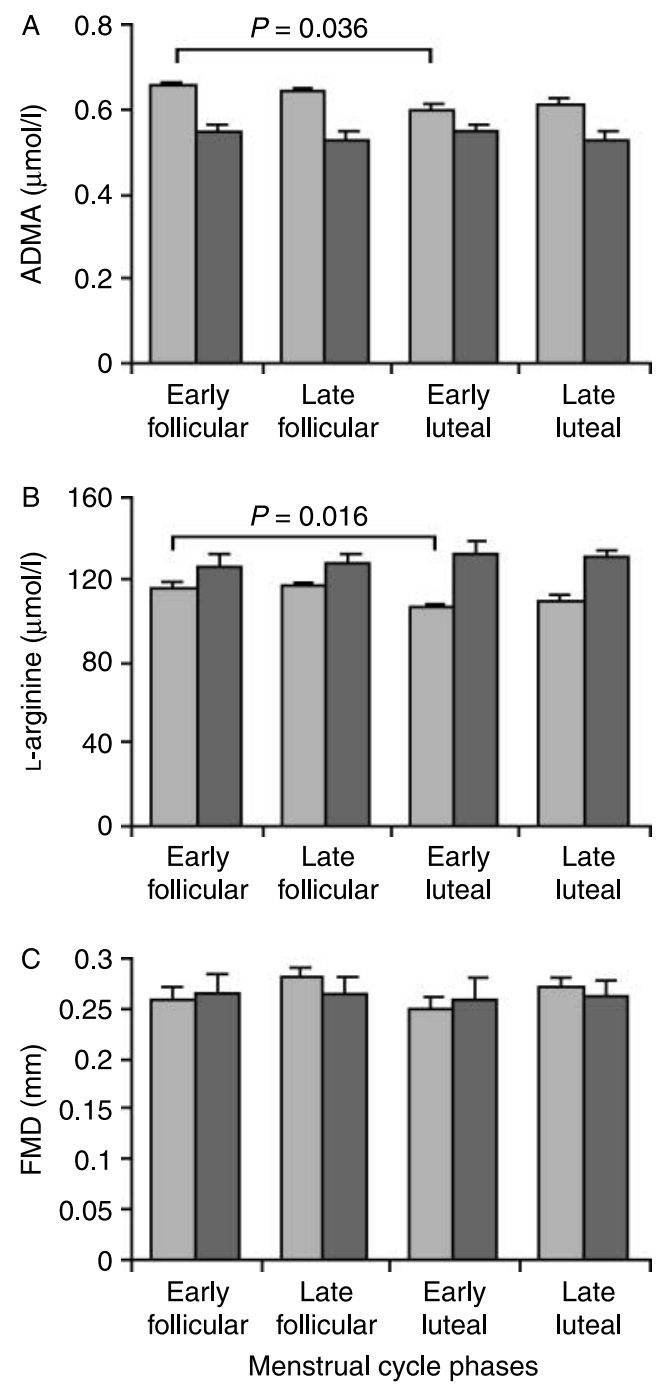

Figure 1 Plasma asymmetric dimethylarginine (ADMA)

(A) and L-arginine (B) concentrations and flow-mediated dilatation (FMD) (C) during different phases of the menstrual cycle in oral contraceptive (OC) users ( $n=318$, dark gray) and non-users $(n=761$, light gray). Data are expressed as mean \pm S.E.M.

\section{The effect of contraceptive type on the dimethylarginine, CRP and FMD}

ADMA concentration was examined according to contraceptive types, and it was noted that ADMA levels were significantly higher $(P=0.009)$ in women using progestin-only $\mathrm{OC}$ than in women using preparations with a constant estrogen dose and low progestin dose (Fig. 3). CRP was two- to three-fold higher in the group using estrogen-containing pills than in their counterparts using progestin-only pills (Fig. 3), and the difference between these groups was significant (median CRP 1.77 vs $0.69 \mathrm{mg} / \mathrm{l}, \mathrm{P}=0.004$ and 2.22 vs 0.69 , $P=0.006)$. The $\mathrm{FMD}^{2}$ was $20 \%$ higher in women in progestin-only group $(10.3 \%)$ than in those receiving estrogen-containing preparations $(8.6 \%)$, but the difference did not achieve statistical significance. Waist circumference of study subjects was not statistically different between different contraceptive type groups $(P=0.118)$.

\section{Relationship between dimethylarginine concentrations and FMD parameters}

Both OC and non-OC users exhibited a significant negative correlation between ADMA and FMD $(r=-0.101, P=0.007$ and $r=-0.139, P=0.016$ respectively) and $\mathrm{FMD} \%(r=-0.088, P=0.019$ and $r=-0.142, P=0.013$ respectively). Accordingly, SDMA had a significant negative correlation with
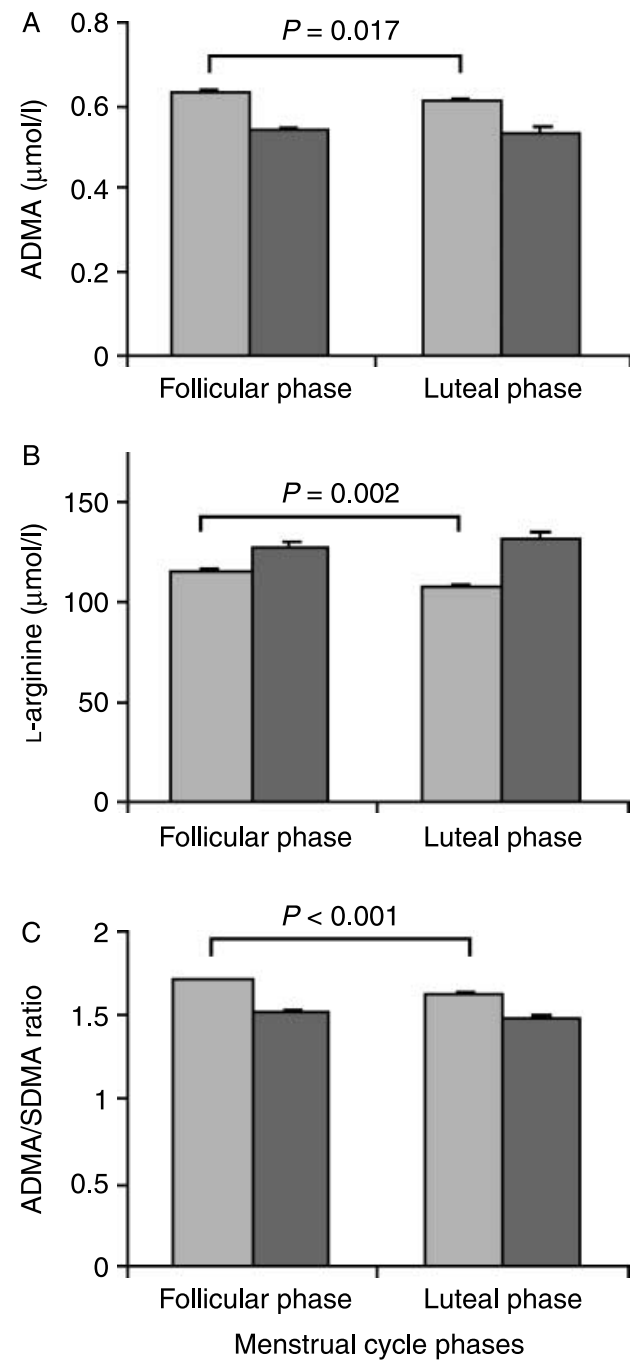

Figure 2 Plasma concentrations of asymmetric dimethylarginine (ADMA) (A) and L-arginine (B), and ADMA/SDMA ratio (C) according to whether women were in the follicular $(n=497)$ and luteal phases $(n=486)$ of the menstrual cycle with (dark gray) and without oral contraceptive (OC) use (light gray). Data are expressed as mean \pm S.E.M. SDMA, symmetric dimethylarginine. 

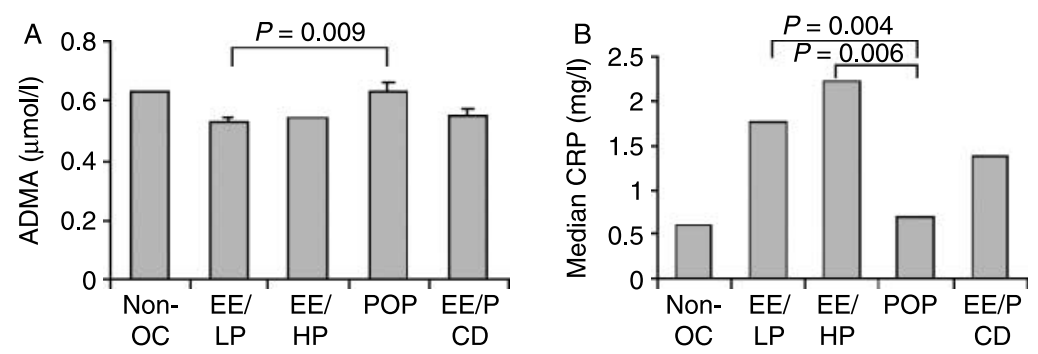

Figure 3 Plasma asymmetric dimethylarginine (ADMA) (A) and C-reactive protein (CRP) (B) concentrations in women using different types of oral contraceptives. Contraceptive types were constant estrogen dose with low progestin dose (EE/LP, $n=110)$, constant estrogen dose with high progestin dose (EE/HP, $n=120$ ), progestin-only contraceptive pills (POPs, $n=10$ ), and combination preparation with changing doses (EE/P CD, $n=68$ ). Data for ADMA are expressed as mean \pm s.E.M., and for CRP they are expressed as median values.

FMD $(r=-0.113, P=0.003)$ and $\operatorname{FMD} \%(r=-0.104$, $P=0.006)$ in non-OC users.

When examining associations during the different phases of the menstrual cycle, only in the early follicular phase was there a significant negative correlation between ADMA and FMD $(r=-0.275, P=0.001)$ and FMD $\%(r=-0.280, P<0.001)$ and also between SDMA and FMD $(r=-0.186, P=0.022)$ and FMD $\%$ $(r=-0.202, P<0.013)$ in non-OC users. In OC users, we did not detect any correlations between ADMA and FMD during the different menstrual cycle phases.

\section{Discussion}

We found that the ADMA concentration varied at different phases of the menstrual cycle in women not using hormonal contraception, whereas among OC users, its levels remained stable. Interestingly, the type of contraception made a substantial difference, since estrogen-containing pills lowered the ADMA concentration significantly in comparison with non-OC users. In contrast, in women using progestin-only contraceptive pills, the ADMA concentration remained at the same level as that encountered in non-OC users. It was also noted that although the overall effect of OC decreased the ADMA concentration, the CRP appeared to be higher in OC users though we detected no significant net effect on FMD.

In vascular cells, estradiol stimulates the expression of endothelial and inducible NOS, leading to NO production and NO-dependent vasodilation (1). In addition, the results from a previous study indicated that NO fluctuates during a natural menstrual cycle. Giusti et al. (22) have demonstrated that the NO concentration was significantly higher in the follicular phase than in the luteal phase. However, Teran et al. (23) did not observe any significant differences between the circulating plasma levels present in the preovulatory and the mid-luteal phase. In this study, we found that the ADMA concentration varied across the menstrual cycle in women not using hormonal contraception, with the level being highest during the early follicular phase of menstrual cycle when estrogen level is low, and lowest in the early luteal phase when the estrogen and progesterone levels are usually high. The difference in ADMA levels between cycle phases was, however, in the order of $4 \%$ only, and it hardly accounts for major biological sequelae. Contraceptive use abolished normal hormonal changes, and no significant variation in the ADMA or L-arginine concentrations was present in women using OC. The differences between the synthetic and natural steroids may have a role to play herein. Interestingly, SDMA, the isomer of ADMA, did not exhibit any variations across the menstrual cycle. This may be due to the fact that SDMA is not as sensitive to hormonal changes because it is eliminated by urinary excretion and not by enzymatic conversion by DDAH into ADMA.

OC use and orally administered HRT lower ADMA and SDMA concentrations either in the combination of estrogen and progesterone or as estrogen alone. In addition, the delivery method for the exogenous estrogen is important since transdermally administered estrogen affects ADMA levels less than orally administered estrogen (24). Accordingly, Holden et al. (25) reported that 2 weeks after subcutaneous implantation of estradiol, there was a significant decrease in ADMA levels. Recently, Bunck et al. (26) reported that both oral and transdermal estrogen therapy in male-to-female transsexuals decreased circulating ADMA. Furthermore, Kilic et al. (27) reported that $50 \mu \mathrm{g}$ transdermal estradiol prevented the increase in ADMA concentration following ovariectomy.

In our study, the comparison of the different OC preparations suggested that it was the exogenous estrogen in the combination of progesterone which decreased ADMA significantly, since in users of progesterone-only pills, ADMA remained at the same level as in non-OC users. However, the group of progestin users was very small, and women who were not willing or unable to use estrogen treatment may have been overrepresented since progestin-only pills may have been prescribed to overweight women or to women suffering from arterial hypertension or those at increased risk for venous thromboembolism or 
cardiovascular diseases. In our study, however, there were no significant differences in the body weights between the different contraception type groups.

Wander et al. (28) reported that endogenous estrogen was associated with a decrease in CRP levels, and endogenous progesterone with an increase in CRP levels. Interestingly, some studies have suggested that exogenous estrogen can increase CRP $(29,30)$, whereas exogenous progesterone decreases CRP (31). However, we did not find any significant differences in CRP levels between different cycle phases in women with or without OC use, a result which is in accordance with the study of Wunder et al. (32). Neither did we find any statistically significant differences in FMD parameters across menstrual cycle phases in normal women.

There are some limitations to our study. First, we did not measure estrogen or progesterone concentrations during menstrual cycle phases to confirm the hormonal status. However, the participants had normal menstrual cycles, and women with an abnormally long cycle (over 40 days) were excluded from the study. Such selection of patients aimed at minimizing the bias produced by overlapping menstrual cycle phases in women without OC use. Secondly, cross-sectional studies are carried out at one time point and changes over time cannot be assessed. However, this study provided information on the normal female population subdivided according to their phase of the menstrual cycle and the effects of the cycle on L-arginine and dimethylarginine concentrations. Many studies on the effect of the menstrual cycle on ADMA, inflammation markers, or FMD have been rather small, and though population-based studies to find reference intervals for ADMA may have been large, they may not have taken into account the menstrual cycle phase of the participating women or else their subjects may have been over 50 years old (33, 34). Blackwell et al. (35) have studied biological variation of ADMA and found that ADMA and SDMA exhibited low intra-individual biological variation (7.4 and $5.8 \%$ respectively), but there were only six women $(n=12)$ in that study and no information on menstrual cycle phase was given.

In conclusion, the hormonal changes during the menstrual cycle appear to have an impact on ADMA concentrations, and therefore, they should be controlled for in future ADMA studies in women of reproductive age. On the contrary, FMD seems to be insensitive to hormonal changes in normal young women, and thus FMD may be measured during any phase of menstrual cycle. In women using estrogen-containing OC preparations, the ADMA concentrations were stable and significantly lower in OC users than in non-OC users. A similar effect has been observed previously in OC-using women affected by PCOS, but not in healthy individuals with no known risks of endothelial dysfunction. Progestin-only pills appeared to result in an ADMA and CRP profile that was very different from that observed in users of combined hormone preparations.

\section{Declaration of interest}

We have no conflict of interest that could be perceived as prejudicing the impartiality of the research reported.

\section{Funding}

This study was financially supported by grants from Kuopio University Hospital (grant no. 15863 and EVO grant nos 5190 and 5031320), EVO grants from Turku University Central Hospital and Academy of Finland (grant nos 77841 and 210283), Social Insurance Institution, the Finnish Foundation for Cardiovascular Research and the Finnish Cultural Foundation, North Savo Regional fund.

\section{References}

1 Mendelsohn ME. Protective effects of estrogen on the cardiovascular system. American Journal of Cardiology $2002 \mathbf{8 9} 12 \mathrm{E}-17 \mathrm{E}$ (discussion 17E-18E).

2 Cevik D, Unay O, Durmusoglu F, Yurdun T \& Bilsel AS. Plasma markers of NO synthase activity in women after ovarian hyperstimulation: influence of estradiol on ADMA. Vascular Medicine 200611 7-12.

3 Ardigo D, Stuehlinger M, Franzini L, Valtuena S, Piatti PM, Pachinger O, Reaven GM \& Zavaroni I. ADMA is independently related to flow-mediated vasodilation in subjects at low cardiovascular risk. European Journal of Clinical Investigation 200737 263-269.

4 Juonala M, Viikari JS, Alfthan G, Marniemi J, Kähönen M, Taittonen L, Laitinen T \& Raitakari OT. Brachial artery flowmediated dilation and asymmetrical dimethylarginine in the Cardiovascular Risk in Young Finns Study. Circulation 2007116 1367-1373.

5 Vallance P. Importance of asymmetrical dimethylarginine in cardiovascular risk. Lancet 2001358 2096-2097.

6 Valkonen VP, Päivä H, Salonen JT, Lakka TA, Lehtimäki T, Laakso J \& Laaksonen R. Risk of acute coronary events and serum concentration of asymmetrical dimethylarginine. Lancet 2001 358 2127-2128.

7 Cooke JP. NO and angiogenesis. Atherosclerosis 20034 53-60.

8 Zoccali C, Bode-Böger S, Mallamaci F, Benedetto F, Tripepi G, Malatino L, Cataliotti A, Bellanuova I, Fermo I, Frölich J \& Böger R. Plasma concentration of asymmetrical dimethylarginine and mortality in patients with end-stage renal disease: a prospective study. Lancet $20013582113-2117$.

9 Ozgurtas T, Oktenli C, Dede M, Tapan S, Kenar L, Sanisoglu SY, Yesilova Z, Yenen MC, Erbil MK \& Baser I. Metformin and oral contraceptive treatments reduced circulating asymmetric dimethylarginine (ADMA) levels in patients with polycystic ovary syndrome (PCOS). Atherosclerosis $2008200336-344$.

10 Savvidou MD, Hingorani AD, Tsikas D, Frölich JC, Vallance P \& Nicolaides KH. Endothelial dysfunction and raised plasma concentrations of asymmetric dimethylarginine in pregnant women who subsequently develop pre-eclampsia. Lancet 2003 361 1511-1517.

11 Beltowski J \& Kedra A. Asymmetric dimethylarginine (ADMA) as a target for pharmacotherapy. Pharmacological Reports $2006 \mathbf{5 8}$ 159-178.

12 Wahbi N, Dalton RN, Turner C, Denton M, Abbs I \& Swaminathan R. Dimethylarginines in chronic renal failure. Journal of Clinical Pathology 200154 470-473.

13 Verhoeven MO, Hemelaar M. Teerlink T, Kenemans P \& van der Mooren MJ. Effects of intranasal versus oral hormone therapy on asymmetric dimethylarginine in healthy postmenopausal women: a randomized study. Atherosclerosis 2007195 181-188.

14 Post MS, Verhoeven MO, van der Mooren MJ, Kenemans P, Stehouwer CD \& Teerlink T. Effect of hormone replacement therapy on plasma levels of the cardiovascular risk factor 
asymmetric dimethylarginine: a randomized, placebo-controlled 12 -week study in healthy early postmenopausal women. Journal of Clinical Endocrinology and Metabolism 200388 4221-4226.

15 Teerlink T, Neele SJ, de Jong S, Netelenbos JC \& Stehouwer CD. Oestrogen replacement therapy lowers plasma levels of asymmetrical dimethylarginine in healthy postmenopausal women. Clinical Science 2003105 67-71.

16 Valtonen P, Laitinen T, Lyyra-Laitinen T, Raitakari OT, Juonala M, Viikari JSA, Heiskanen N, Vanninen E, Punnonen K \& Heinonen S. Serum L-homoarginine concentration is elevated during normal pregnancy and is related to flow-mediated vasodilatation. Circulation Journal 200872 1879-1884.

17 Holden DP, Fickling SA, Whitley GS \& Nussey SS. Plasma concentrations of asymmetric dimethylarginine, a natural inhibitor of nitric oxide synthase, in normal pregnancy and preeclampsia. American Journal of Obstetrics and Gynecology 1998 178 551-556.

18 Raitakari OT, Juonala M, Rönnemaa T, Keltikangas-Järvinen L, Räsänen L, Pietikäinen $M$, Hutri-Kähönen N, Taittonen L, Jokinen E, Marniemi J, Jula A, Telama R, Kähönen M, Lehtimäki T, Åkerblom HK \& Viikari JSA. Cohort Profile: the Cardiovascular Risk in Young Finns Study. International Journal of Epidemiology 200837 1220-1226.

19 Juonala M, Viikari JS, Hutri-Kähönen N, Pietikäinen M, Jokinen E, Taittonen L, Marniemi J, Rönnemaa T \& Raitakari OT. The 21-year follow-up of the Cardiovascular Risk in Young Finns Study: risk factor levels, secular trends and east-west difference. Journal of Internal Medicine 2004255 457-468.

20 Teerlink T, Nijveldt RJ, de Jong S \& van Leeuwen PA. Determination of arginine, asymmetric dimethylarginine, and symmetric dimethylarginine in human plasma and other biological samples by high-performance liquid chromatography. Analytical Biochemistry 2002303 131-137.

21 Cockcroft DW \& Gault MH. Prediction of creatinine clearance from serum creatinine. Nephron 197616 31-41.

22 Giusti M, Fazzuoli L, Cavallero D \& Valenti S. Circulating nitric oxide changes throughout the menstrual cycle in healthy women and women affected by pathological hyperprolactinemia on dopamine agonist therapy. Gynecological Endocrinology 200216 407-412.

23 Teran E, Escudero C \& Vivero S. Physiological changes in platelet aggregation and nitric oxide levels during menstrual cycle in healthy women. Nitric Oxide 20027 217-222.

24 Verhoeven MO, Hemelaar M, van der Mooren MJ, Kenemans P \& Teerlink T. Oral, more than transdermal, oestrogen therapy lowers asymmetric dimethylarginine in healthy postmenopausal women: a randomized, placebo-controlled study. Journal of Internal Medicine 2006259 199-208.

25 Holden DP, Cartwright JE, Nussey SS \& Whitley GS. Estrogen stimulates dimethylarginine dimethylaminohydrolase activity and the metabolism of asymmetric dimethylarginine. Circulation 2003 $1081575-1580$.
26 Bunck MC, Giltay EJ, Diamant M, Gooren LJ \& Teerlink T. Differential effects of cross-sex hormonal treatment on plasma asymmetric dimethylarginine (ADMA) in healthy maleto-female and female-to-male transsexuals. Atherosclerosis 2009 $206245-250$.

27 Kilic S, Yilmaz N, Erdogan G, Aydin M, Tasdemir N, Doganay M \& Batioglu S. Effect of non-oral estrogen on risk markers for metabolic syndrome in early surgically menopausal women. Climacteric 2009 DOI: 10.1080/13697130902914650.

28 Wander K, Brindle E \& O'Connor KA. C-reactive protein across the menstrual cycle. American Journal of Physical Anthropology 2008 $136138-146$

29 Raitakari M, Mansikkaniemi K, Marniemi J, Viikari JSA \& Raitakari OT. Distribution and determinants of serum highsensitive C-reactive protein in a population of young adults. The Cardiovascular Risk in Young Finns Study. Journal of Internal Medicine 2005258 428-434.

30 Haarala A, Eklund C, Pessi T, Lehtimäki T, Huupponen R, Jula A, Viikari JSA, Raitakari $\mathrm{O}$ \& Hurme $\mathrm{M}$. Use of combined oral contraceptives alters metabolic determinants and genetic regulation of C-reactive protein. The Cardiovascular Risk in Young Finns Study. Scandinavian Journal of Clinical and Laboratory Investigation 200969 168-174.

31 Skouby SO, Gram J, Andersen LF, Sidelmann J, Petersen KR \& Jespersen J. Hormone replacement therapy: estrogen and progestin effects on plasma C-reactive protein concentrations. American Journal of Obstetrics and Gynecology $2002 \mathbf{1 8 6}$ 969-977.

32 Wunder DM, Yared M, Bersinger NA, Widmer D, Kretschmer R \& Birkhauser MH. Serum leptin and C-reactive protein levels in the physiological spontaneous menstrual cycle in reproductive age women. European Journal of Endocrinology $2006 \mathbf{1 5 5}$ $137-142$.

33 Schulze F, Maas R, Freese R, Schwedhelm E, Silberhorn E \& Böger RH. Determination of a reference value for $N(G)$, $N(G)$-dimethyl-L-arginine in 500 subjects. European Journal of Clinical Investigation $200535622-626$.

34 Teerlink T. HPLC analysis of ADMA and other methylated L-arginine analogs in biological fluids. Journal of Chromatography. B $200785121-29$.

35 Blackwell S, O'Reilly DS \& Talwar D. Biological variation of asymmetric dimethylarginine and related arginine metabolites and analytical performance goals for their measurement in human plasma. European Journal of Clinical Investigation 200737 364-371.

Received 29 October 2009

Accepted 23 November 2009 